\title{
Validation and optimization of BIANCA for the automatic segmentation of extensive white matter hyperintensities
}

\author{
Yifeng Ling1,3, Eric Jouvent ${ }^{1,2}$, Louis Cousyn1 ${ }^{1}$, Hugues Chabriat' ${ }^{1,2}$, François De Guio ${ }^{1,2}$ \\ 1 University Paris Diderot, Sorbonne Paris Cité, UMR-S 1161 INSERM, F-75205 Paris, France \\ 2 AP-HP, Lariboisière Hosp, Department of Neurology, F-75475 Paris, France, and DHU NeuroVasc Sorbonne Paris Cité, Paris, France; \\ ${ }^{3}$ Huashan Hospital, Department of Neurology, Fudan University, Shanghai, China
}

\section{Introduction}

White matter hyperintensities (WMH) are a hallmark of small vessel diseases (SVD). Yet, no automated segmentation method is readily and widely used, especially in patients with extensive WMH where lesions are close to the cerebral cortex. BIANCA (Brain Intensity AbNormality Classification Algorithm) is a new fully automated, supervised method for WMH segmentation. In this study, we optimized and compared BIANCA against a reference method with manual editing in a cohort of CADASIL patients with extensive WMH.

\section{Methods}

This was achieved in two datasets: a clinical protocol with 90 patients having 2-dimensional FLAIR and an advanced protocol with 66 patients having 3dimensional FLAIR. We first determined simultaneously which input modalities (FLAIR alone or FLAIR + T1) and which training sets were better compared to the reference. Three strategies for the selection of the threshold that is applied to the probabilistic output of BIANCA were then evaluated. Accuracy of the segmentation was assessed through measures of spatial agreement and volumetric correspondence with respect to reference segmentation.
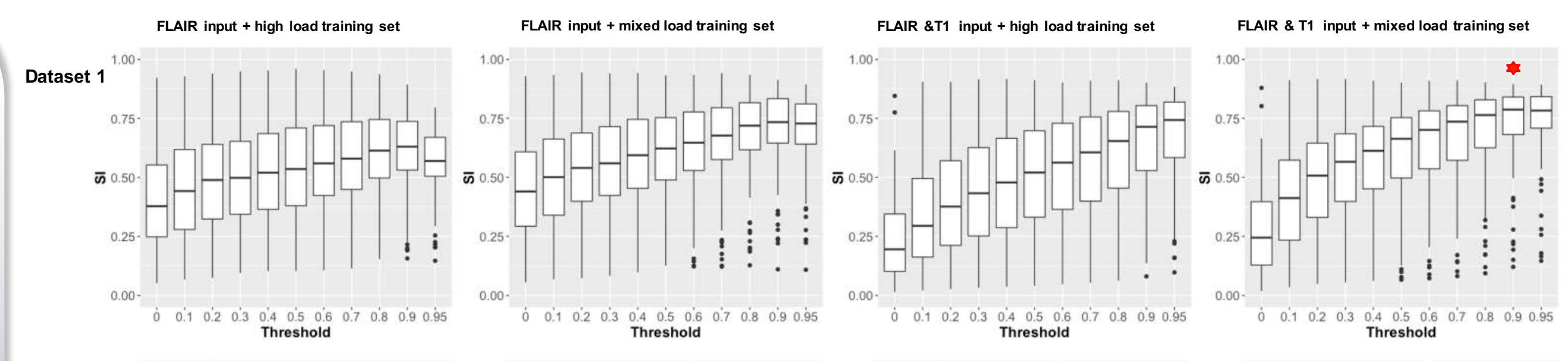

Dataset 2

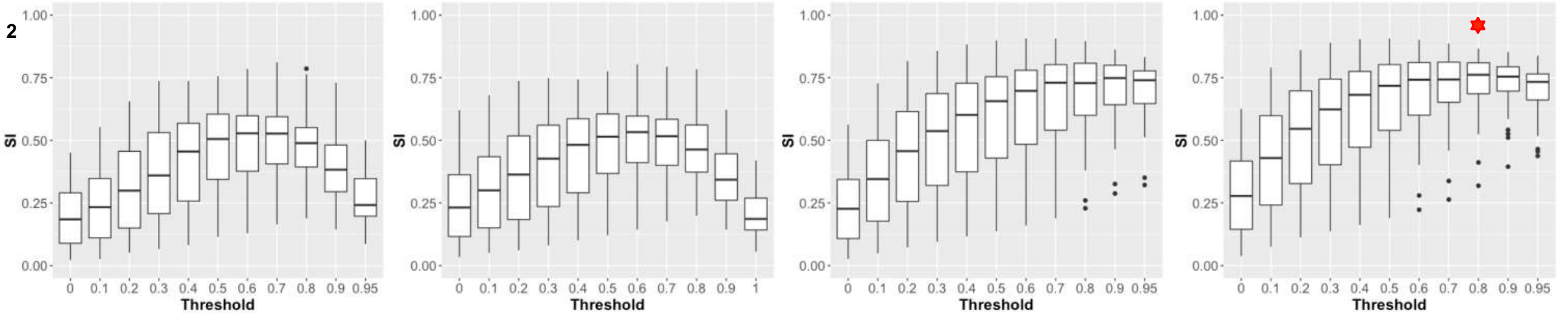

Fig. 1 Box plots of SI for different thresholds for all tests of BIANCA optimization phase I. The plots show the similarity index (SI) used to evaluate BIANCA performance using different thresholds (x axis). Red stars indicate the best combination of options chosen for each dataset.
Table. 1 Comparison of BIANCA performance for the different strategies for threshold selection

\begin{tabular}{lllllllll}
\hline Datasct & Threshold sclection & No, of patients & Best threshold & SI (IQR) & FPR (IQR) & FNR (IQR) & ICC & CV, \% \\
\hline Datasct 1 & Overall & 90 & 0.9 & $0.79(0.68-0.84)$ & $0.23(0.18-0.43)$ & $0.14(0.10-0.21)$ & 0.81 & 15.4 \\
& On Fazekas Scale (H/L)* & $43 / 47$ & $0.9 / 0.95$ & $0.79(0.71-0.84)$ & $0.22(0.16-0.39)$ & $0.18(0.11-0.24)$ & 0.86 & 12.5 \\
& Individual & 90 & $0.95(0.9-0.95)^{* *}$ & $0.80(0.72-0.85)$ & $0.21(0.16-0.37)$ & $0.15(0.12-0.21)$ & 0.97 & 6.8 \\
Dataset 2 & Overall & 66 & 0.8 & $0.76(0.69-0.81)$ & $0.20(0.10-0.36)$ & $0.22(0.16-0.28)$ & 0.78 & 30.1 \\
& On Fazekas Scale (H/L)* & $40 / 26$ & $0.7 / 0.9$ & $0.78(0.72-0.81)$ & $0.23(0.12-0.28)$ & $0.23(0.14-0.31)$ & 0.87 & 11.1 \\
& Individual & 66 & $0.8(0.6-0.9)^{* *}$ & $0.80(0.75-0.83)$ & $0.20(0.17-0.24)$ & $0.20(0.16-0.27)$ & 0.98 & 3.5 \\
\hline
\end{tabular}

* $H$, high WMH load (Fazekas scales 4-6); L, low WMH load (Fazekas scales <4)

**median (IQR)

$S I$, Dice similarity index; $F P R$, false positive ratio, $F N R$, false negative ratio; $I C C$, intraclass correlation coefficient; $C V$, coefficient of variation; $I Q R$, interquartile range

\section{Conclution}

Our results suggest that BIANCA is a reliable and fast automatic segmentation method to extract masks of $\mathrm{WMH}$ in patients with extensive lesions.

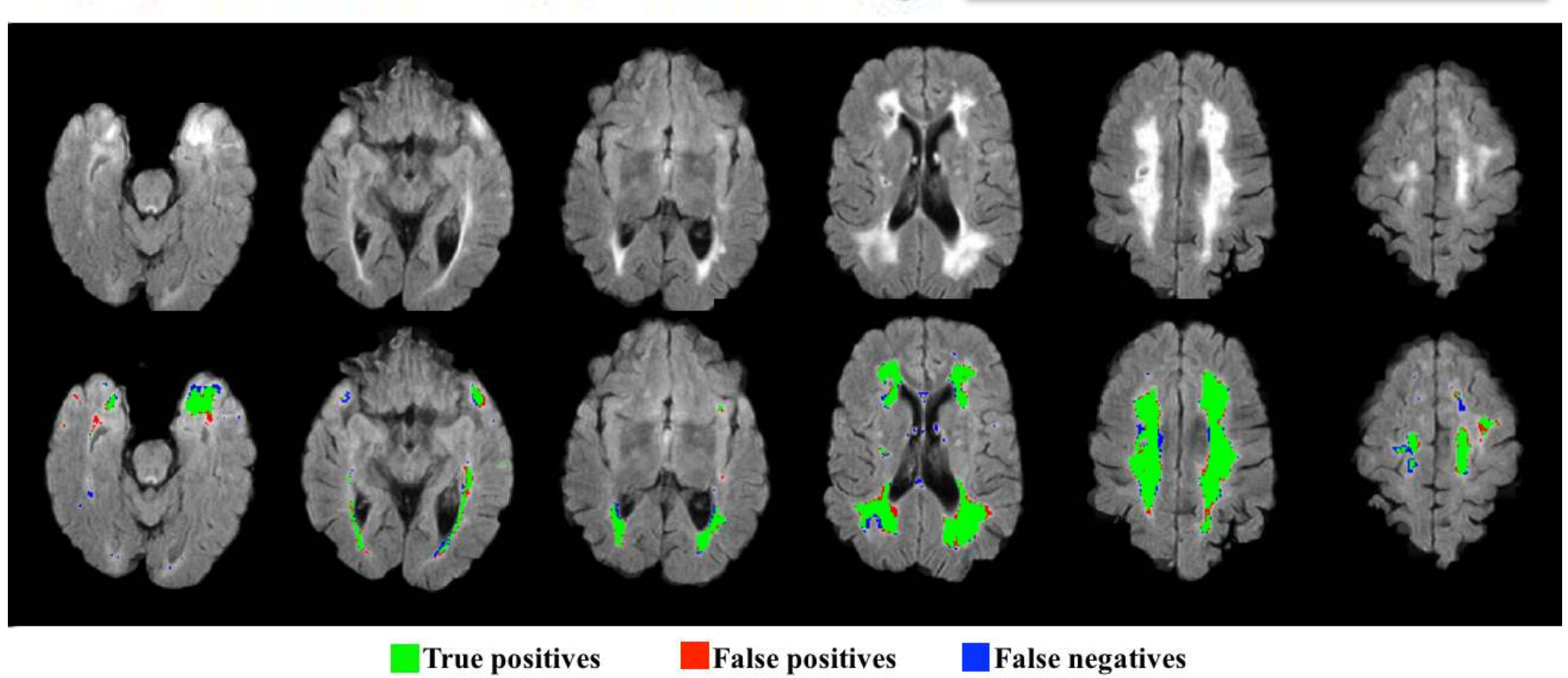

Fig. 2 Comparison of BIANCA results and reference segmentation with the best input modality and training set
Based on all our tests, we identified multimodal inputs $($ FLAIR + T1), mixed WMH load training set and individual threshold selection as the best conditions to automatically segment WMH in our cohort. A median Dice similarity index of $0.80(0.80)$ and an intraclass correlation coefficient of $0.97(0.98)$ were obtained for the clinical (advanced) protocol. 